\title{
The Effective Sleep Scheduling in Wireless Opportunistic Networks
}

\author{
Wenzao Li ${ }^{1}$, Zhan Wen ${ }^{1, \text { a }}$,Jianwei Liu ${ }^{1}$, Shuang Xiao ${ }^{1}, \mathrm{Xi} \mathrm{Wu}^{2}$, Yue $\mathrm{Cao}^{3}$, Jiliu Zhou ${ }^{2}$ \\ ${ }^{1}$ College of Communication Engineering, Chengdu University of Information Technology, Chengdu 610225, China \\ ${ }^{2}$ School of Computer Science, Chengdu University of Information Technology, Chengdu 610225, China \\ ${ }^{3}$ Department of Computer and Information Sciences, Northumbria University, Newcastle, UK
}

\begin{abstract}
One of the purposes of Internet of Things (IoT) is to reach more deeper perception. For this purpose, the efficient energy consumption is necessary among intelligent devices that make up the part of Opportunistic Networks (ONs). It is irrational for an ONs without any sleep scheduling because of awful user experience. We explore a sleeping schedule which is based on duty cycling for mobile devices to reduce energy consumption of ONs. To see how schedule affects the performance of ONs, we took a series of simulations and the results indicated that the sleeping schedule is an efficient method for prolonging the network life time in ONs. The successful delivery ratio can increase two to three times when factor $\mathrm{T}_{\mathrm{r}}$ equal 0.2 . We also observed that the network matrices are acceptable, and the network survival time can be extended effectively in ONs.
\end{abstract}

\section{Introduction}

Recently, various of intelligent devices (mobile phone, smart bands or tablets) widespread appear in our daily life. And these devices are equipped short-range wireless communication module. Therefore it can provide the enhanced wireless communication ability for different application systems[1]. ONs are based on Delay Tolerant Networks (DTNs) technology. It consists of sensors with short-range communication ability, the message transmits by store-and it attracts many researches in the context of Device-to-Device(D2D) communication in 5G[2, 3]. Especially, city areas become important places of data generation and consumption. In social environments, ONs network can enrich the integrated ecosystem which is create by IoT technology[4]. The ubiquitous wireless application systems play pivotal roles in Smart City, which is established by various of devices such as smart vehicles, personal electronics and wireless infrastructures. These devices always carried by human so they were given the mobility feature. Most of mobile devices exchange the message by encounter devices nearby. Therefore, the mobile devices can enhance the performance of seamless connectivity among data islands and depth of perceptive information in IoT. Then, many mobile sensor networks applied mobile devices or vehicles to build for emerging applications and data collection system. So, an obvious question arises, the energy of mobile devices is always supplied by battery, and the energy is limited during a period of time. But obviously, ONs communication network structure includes a number of mobile devices. These devices run numerous programs and own various battery specifications. It is difficult to estimate the energy efficiency due to complex power supply conditions. The main devices in IoT technology are various portable devices, which have been performed for many applications and services. And these devices are used for not only one task in our daily life, then the energy consumption for short-distance communication is ambiguity for users. To assess and optimize the network energy efficiency, the energy consumption model and a sleep scheduling are needed in such complex situation.

With the consideration to the network level and energy efficiency in ONs, the main research contributions are listed as follows:

(1) The energy consumption model in opportunistic translation is described.

(2) The network lifetime and network performances are effective promote by a sleep scheduling which is usually discussed as duty cycle in WSN.

The rest of this paper is organized as follows. Section 1 describes the related work. Then we describe the energy consumption model for the device to transmit messages to another device nearby in ON networks in section 2. The section 3 gives the simulation environment and results. Finally, we give a conclusion in section 4 .

\section{Related Works}

Generally speaking, mobile devices are mainly various smart phones or other portable equipments. The mobile devices need neighbor scanning and transmitting

\footnotetext{
${ }^{a}$ Corresponding author:Zhan Wen wenzhan@cuit.edu.cn
} 
continuously in ONs. However, the energy consumption of mobile devices depends on the encounter opportunity. Obviously, contact discovery need a response message in the routing process, and this phenomenon is frequently happened in high node density areas. The massive multi-hop transmitting and transmitting among devices will lead to excessive energy consumption, which will give participants some unpleasant experience. In such case, the energy efficiency should be considered in ONs. In ONs, the energy consumption mainly occurs from scan device nearby, scan response behavior and transmitting messages[5]. Y. Dou describe a contact model[6] which sets the contact probing interval for the energy conservation. But the energy efficiency depends on the routing method, thus the scanning factor was ignored under the circumstance. In literature[7], B.J. Choi also described the energy saving mechanism. The fundamental problem is that the listening mode spend more than $95 \%$ percent of total energy for neighbor searching. Thus, most of the energy consumption is not efficient for the data transition. Generally, a sleep schedule includes wakeup method. For example, mobile nodes may equip low power radio module to wake up the device, named nodes. As the well-known IEEE 802.11 PSM protocol[8], can make the mobile nodes turn on and turn off for energy saving. It can be described as duty cycling in such single hop networks. The sleeping behaviors depend on the prediction of contact opportunity. Zeng F. et al.[9] also focus on the listening behavior and propose an adaptive scheduling mechanism which based on self-similarity feature. But these adaptive duty-cycling methods should be automatic synchronized by global clock and the inter-contact intervals of pair node. In precious research, the part of scan energy is the largest proportion in ONs, then the sleeping scheduling can prolong the network life time. Also, the message transmission can consume the nodes' limited energy, the literature[10,11] also discuss routing method for energy consumption. They limited the message copies in DTN routing, and then energy conservation is achieved by this way. The human-carried communication devices, which mainly consist of ONs in urban scenario, always take on variety of application tasks such as navigation, socializing, and etc. Generally, the inefficient energy consumption of devices will decrease the user experience although each device has opportunities for energy replenishment in human daily life. The ONs, as a data transmission way, is a function for the mobile intelligent device. Thus, the energy of ONs should be used as little as possible due to the limited electric capacity[12]. The ONs always be structured in urban areas which is different from other sensing areas. Hence the energy saving mechanism is increasingly important in urban based ONs. From the above, this paper presents the duty-cycling based sleeping schedule for ONs in urban environments that aims to improve the energy efficiency. This sleeping scheduling not only prolongs the network lifetime but also gives benchmarks for follow-up sleeping strategy.

\section{Duty-cycling Method for ONs in Urban Areas}

The common discussed duty-cycling method is widely used in Wireless Sensor Networks (WSN) because of the limited energy of nodes. The sleeping scheduling aims to improve the energy efficiency and extend the survival time of nodes. ONs are widely discussed with the rapid development of the personal intelligent mobile devices. The main different between WSN and ONs is the mobility of nodes. Generally, node carries the packets at the moving status and transmits the packets during the encounter opportunities in ONs. Thus, nodes should continue scanning the nodes which is covered by the transmitting range in such multi-hop wireless networks. Because of this working mechanism, the discovering behavior will exhaust the limited energy continuously.

\subsection{Energy Model for ONs}

Generally, the energy model of ONs is described by literature[13]. From the literature, the energy consumption of DTN consists mainly of three parts, scan energy $E_{s}$, scan response energy $E_{\text {res }}$ and transmission energy $E_{t r}$. Then the energy consumption model can be represented as equation (1).

$$
E_{c}=E_{s}+E_{\text {res }}+E_{t r}
$$

As previously mentioned, the $E_{s}$ is a big part of the energy consumption in ONs. The $E_{\text {res }}$ is the energy consumption for that when a node response for a scanning message, thus it depends on the contact probability. Then the inter-contact time is defined to describe the encounter intermittent time of node[14]. Generally, the ONs is a sparse node network, thus the inter-contact time among nodes are relatively long time period. If we assume a time period $t$ for a node $i$. The energy consumption $E_{c}^{(i)}(t)$ can be represented by equation (2):

$$
E_{c}^{(i)}(t)=\frac{t}{t^{\prime}} E_{s}+T_{m}(t) E_{\text {res }}+T_{n}(t) E_{t r}
$$

Where the $t^{\prime}$ presents the scanning interval time. The $T_{m}(t)$ presents the cumulative number of responses to another scanning message during the time period $t$. In a similar way, the $T_{n}(t)$ presents the cumulative number of packet transmission. In the actual situation, the mobile intelligent devices (mobile phone, smart bracelet and etc.) play the role of mobile nodes, which could take on multiple tasks. For instance, the intelligent devices can be used for calls or navigation. Therefore, the energy consumption of ONs is a part of the total energy consumption for a single node. It's a hard problem to estimate the energy consumption due to the using habit, power supplement, computational power and etc. that is to say, the $E_{c}^{(i)} \in S$ where $S$ include the operation system energy consumption, personal application energy consumption and etc. Meanwhile, the energy supplement cannot be foreseen. So, we ignore the standby energy consumption and we use the equation (2) to describe the energy consumption states for ONs in metropolis areas. The mobile node should have continuous scanning for the purpose of neighbor node discovering. The neighbor node will response the discovering signal and it will consumes some transmitting energy. Then, the message will be transmitted after a pair of nodes finish a connection and the node will expend the data transmitting energy which 
present by $T_{n}(t)$. Obvious, in a sparse wireless network, the scan energy spends a large partition of energy $E_{c}$. Therefore, the duty-cycling approach is an effective sleeping scheduling for improving energy efficiency in metropolis.

\subsection{Duty Cycling Method for Energy Saving}

Duty Cycling methods are widely discussed in WSN for energy saving[15]. The sensors are in turn-off and turn-on status. When it is in turn off status, the transceiver of sensor is turn off and the sensor cannot take part in any communication tasks. Generally, the turn-on and turn-off statuses are spread regularly on the time axis. The work duty cycling of a sensor can be described as figure 1 .

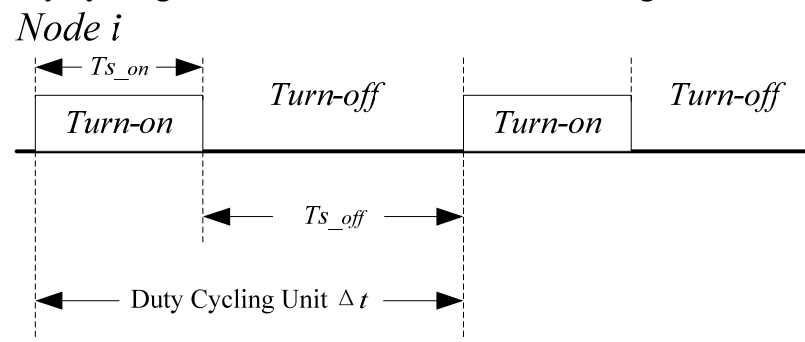

Fig.1 The status of swapping of a node in duty cycling method in WSN

From figure 1, node $i$ switch working status Ts_on time period and $T s_{\text {off }}$ time period for the purpose of prolonging the node's survival time. The method can reduce the energy consumption obviously. The working duty ratio means how long can it works in each time period $\Delta t$. And there is adaptive duty cycling method for energy saving[16], it should have a node wake-up mechanism.

\section{Duty Cycling as Sleeping Schedule in ONs}

The main difference between WSN and ONs is that nodes have mobility in opportunistic networks. Therefore, duty cycling method should face some roadblocks. (1) There is no timely information can be received for entire network, thus the turn-on and turn-off status should consider the clock synchronization for all nodes. The data transmission required a pair of awake nodes which is in each communication range. (2) The sleeping schedule should increase the average latency of delivery because of the fewer contact times and unforeseeable encounter opportunity in ONs. However, the sleeping schedule is necessary for ONs in urban areas due to some reasons. (1) The ONs main consist of intelligent mobile devices, it is a sparse network essentially. Then, the relatively long inter-contact time surely will bring on superabundant $E_{S}$, which should decrease the energy efficiency and short the survival time of nodes. (2) The delivery ratio is one of most important key metrics in ONs, a suitable efficient sleeping schedule can improve the delivery ratio in limit energy circumstances.

In ONs, the mobile communication devices are carried by person or assembled in car. Then, the mobility pattern has social characteristics, the nodes will have longer moving pause periods. For instances with this, people rest at home or working in office. But the data transmission opportunities are based on the mobility of nodes, it is more necessary to design a sleeping schedule in ONs. It is similar to duty cycling using in WSN, each node has turn-on and turn-off status in ONs. But it is only to application layer in an intelligent device. In order to ensure the contact nodes in the same status, the duty cycling units consider synchronized in entire network.

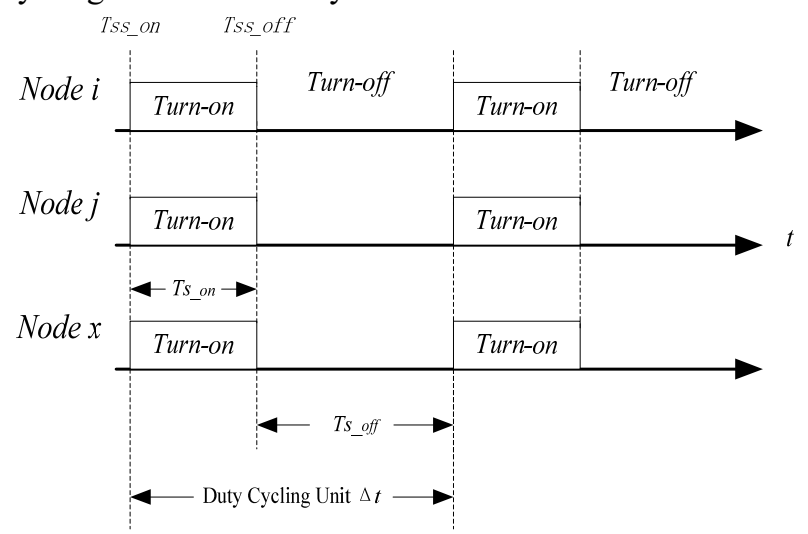

Fig.2 The status of swapping of a node in duty cycling method in ONs

The sleeping time period in ONs are established based on the global clock synchronization in application layer. If the short range communication technology use the Bluetooth, one approach is adopt Bluetooth clock to synchronized the slaves by master or layer- 2 techniques [17, 18]. Each node is awaked during the time point TsS_on and Tss_off. When nodes moving in a communication range, the synchronization statue ensure they are in Turn-on statue. In this sleeping schedule, the mobile nodes will stop neighbor searching during Ts_off time period. And the duty cycle $T_{r}$ can be calculated by equation (3).

$$
T_{r}=\frac{T s_{\_} o n}{\Delta t}
$$

Where $\Delta t$ equal to Ts_on plus the Ts_off time duration.

\section{Simulation and results}

In order to improve the reality of ONs scenario, we choose the Working Day Movement Model (WDM) and The Opportunistic Network Environment (The ONE) simulator. The Helsinki map is selected for urban areas. In order to observe the effect of this sleeping schedule in ONs, we choose the message flooding method for message routing.

The simulation scenario is established by table 1 .

\begin{tabular}{|c|c|c|}
\hline Groups & Type & Nodes \\
\hline $\mathrm{A}, \mathrm{B}, \mathrm{C}, \mathrm{D}$ & Pedestrian & 40 for each \\
\hline$a, b, c, d$ & Bus & 4 for each \\
\hline $\mathrm{T}, \mathrm{P}, \mathrm{Q}$ & Tram & 2 for each \\
\hline
\end{tabular}

The key parameters of the sleeping schedule are list as table 2 . 
Tab. 2 the key parameters of the sleeping schedule

\begin{tabular}{cc}
\hline Parameter name & Value \\
\hline$\Delta t$ & 5 seconds \\
$E_{s}$ & $1 / 3.0^{*} 10^{5}$ percent \\
$E_{\text {res }}$ & $1 / 3.0^{*} 10^{5}$ percent \\
$E_{\text {res }}$ & $2 / 3.0^{*} 10^{5}$ percent \\
\hline
\end{tabular}

We chose the $T_{r}$ as 1,0.2, 0.4, 0.6 and 0.8 respectively, and duty cycling equal one means the sleeping schedule is invalid. The Epidemic routing is selected for ONs running, because it can transmit messages when nodes encounter in right condition. Then we carried out multiple simulations with the sleeping schedule.

In order to observe energy efficiency, the energy expenditure of nodes and the key network indicators are important for a sleeping schedule. The energy expenditure with different $T_{r}$ in ONs are shown in figure 3.

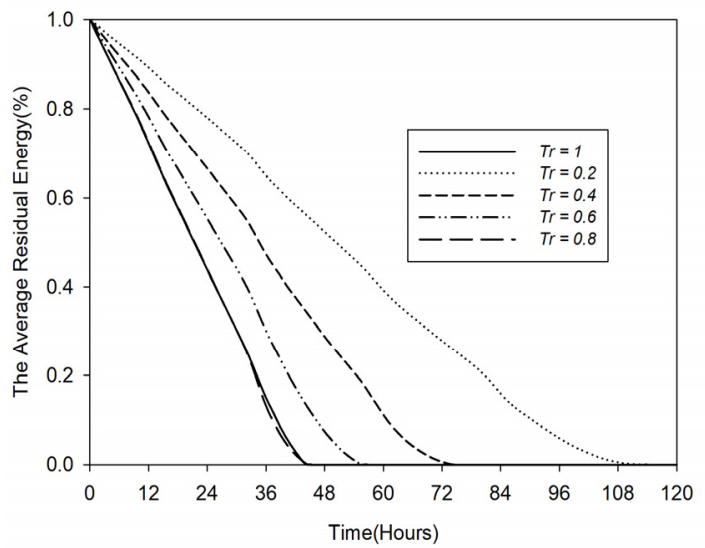

Fig. 3 The energy expenditure in ONs over time

It shows that the 0.2 is the best in energy efficiency. The network exhausts the energy after 108 hours. If the network runs with no any sleeping schedule or working at $0.8 T_{r}$, the network will dead at less than 48 hours. This network energy efficiency is half of $0.2 T_{r}$. The $0.4 T_{r}$ shows the second best in ONs.

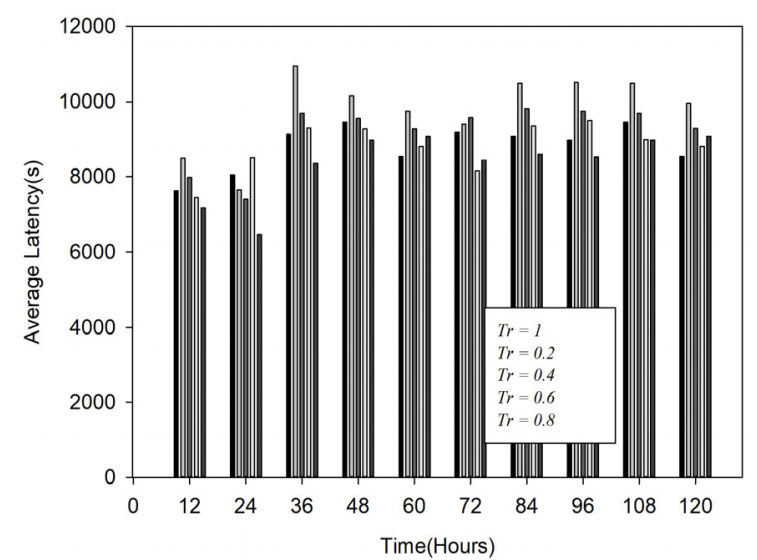

Fig.4 The successful delivery ratio with various $\operatorname{Tr}$ over time

In ONs, the average latency presents the average spend time on successful delivered messages. For a data transmission network, the shorter latency the better application system is based on ONs technology. It is clear in figure 4, the $T r=0.2$ shows the highest latency in this scenario. The 0.8 and 1 show the relatively low latency. It means that nodes in turn-off status result in the message transmitting stagnation.

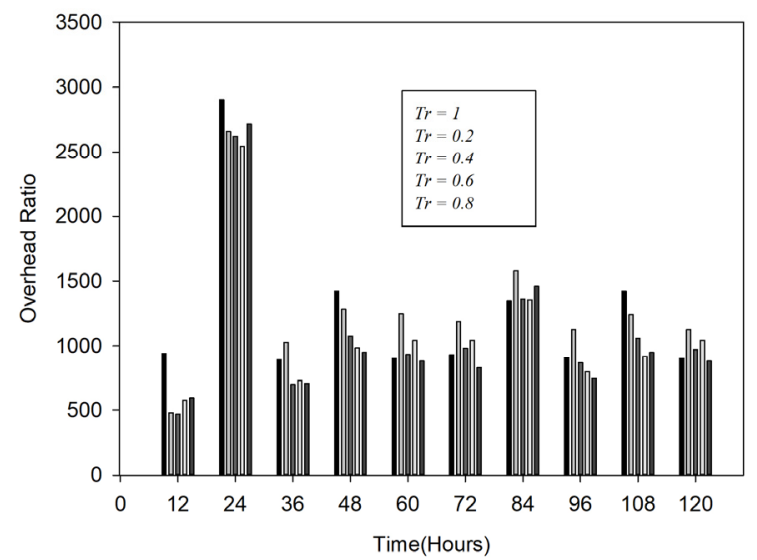

Fig.5 The overhead ratio with various Tr over time

Network overhead ratio can reflect the invalid in ONs. Because it runs with multiple copies routing strategy, the higher value signifies the greater network pressure. It can result the message drop ratio in ONs. In figure 5, the experiment results indicate that the $0.2 \mathrm{Tr}$ is not well, the performance of $0.2 \mathrm{Tr}$ and $1 \mathrm{Tr}$ for overhead ratio is similar. And it is acceptable in ONs.

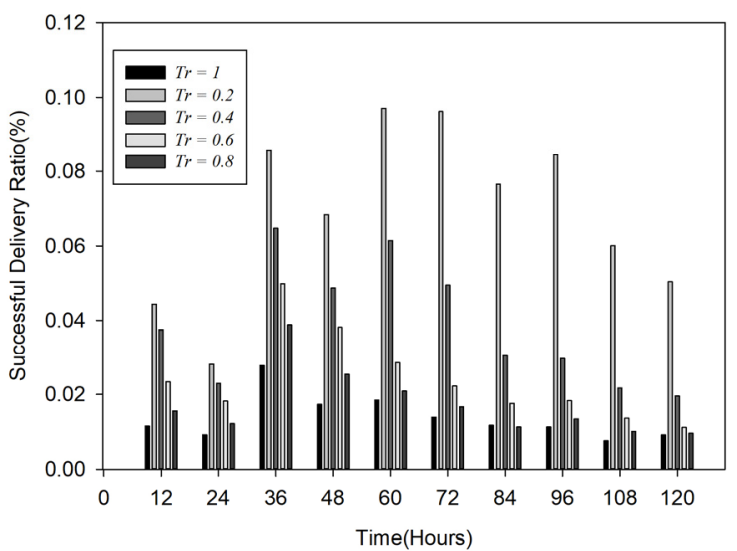

Fig.6 The successful delivery ratio with various $T r$ over time

From figure 6, the $0.2 \operatorname{Tr}$ shows the best performance in successful delivery ability in ONs. $0.4 \mathrm{Tr}$ and $0.6 \mathrm{Tr}$ show that the successful delivery ratio decreased by the more working time in ONs with limited energy. And the sleeping schedule with $0.2 \operatorname{Tr}$ can improve the successful delivery ratio two to three times than $\mathrm{ONs}$ with none sleeping schedule. The results indicate that the suitable sleeping mechanism can increase the delivery ability in mobile sensor networks. The main cause is that ONs is a sparse network in urban areas, thus a lot of energy is used for neighbor scanning. Therefore, in the case of energy constrain, the suitable sleeping schedule can effectively prolong the network life time.

\section{Conclusions}

ONs as one of DTN, it has more application prospects in IoT. It differs from the traditional wireless sensor networks due to the mobility of nodes. Then the sleeping schedule should be redesigned by deployment scenario. 
Duty cycling method has effect in ONs, thus this paper discussed the sleeping schedule based on duty cycle method in ONs. The results indicated that, except the less-than-ideal average latency, the sleeping schedule can optimize the energy consumption and increase the successful delivery ration for ONs in urban areas. It is also important that it can serve as a benchmark for further similar researches.

\section{Conflicts of Interest}

The authors declare that there are no conflicts of interest regarding the publication of this paper.

\section{Acknowledgements}

The authors would like to thank the reviewers for their insightful feedback and valuable suggestions. This work was financially supported by the Science and Technology Department of Sichuan Province, Fund of Science and Technology Planning (No. 2018JY0290).

\section{References}

1. Wu, Y., S. Deng, and H. Huang, Performance analysis of hop-limited epidemic routing in DTN with limited forwarding times. International Journal of Communication Systems, 2015. 28(15): p. 2035-2050

2. Lau, G., et al., Context-aware RAON middleware for opportunistic network. Pervasive \& Mobile Computing, 2017. 41.

3. Dede, J., et al., Simulating Opportunistic Networks: Survey and Future Directions. IEEE Communications Surveys \& Tutorials, 2017.

4. Cuka, M., et al., Implementation and performance evaluation of two fuzzy-based systems for selection of IoT devices in opportunistic networks. Journal of Ambient Intelligence and Humanized Computing, 2018: p. 1-11.

5. Kaur, S., A Review of Energy Consumption on DTN Routing Protocols.

6. Dou, Y., F. Zeng, and W. Li. Energy-Efficient Contact Detection Model in Mobile Opportunistic Networks. in International Conference on Wireless Algorithms, Systems, and Applications. 2017. Springer.

7. Choi, B.J. and X. Shen, Adaptive Asynchronous Sleep Scheduling Protocols for Delay Tolerant Networks. 2011: IEEE Educational Activities Department. 1283-1296.

8. Chumchu, P., An extension to IEEE 802.11 power save mode for NS-3. 2015: p. 799-804.

9. Zeng, F., et al., Efficient Listening and Sleeping Scheduling Mechanism Based on Self-Similarity for Duty Cycle Opportunistic Mobile Networks. Information, 2017. 8(3): p. 87.
10. Li, Y., et al. Optimal Opportunistic Forwarding Policies for Energy-Constrained Delay Tolerant Networks. in IEEE International Conference on Communications. 2010.

11. Li, Y., et al. Performance Evaluation of Routing Schemes for Energy-Constrained Delay Tolerant Networks. in IEEE International Conference on Communications. 2012.

12. Tan, D.N., et al. Mobile charging and data gathering in multiple sink Wireless Sensor Networks: How and why. in International Conference on System Science and Engineering. 2017.

13. Keränen, A., J. Ott, and T. Kärkkäinen. The $O N E$ simulator for DTN protocol evaluation. in International Conference on Simulation TOOLS and Techniques. 2009.

14. Luo, G., et al., Exploiting intercontact time for routing in delay tolerant networks. European Transactions on Telecommunications, 2013. 24(6): p. 589-599.

15. Bonomi, S., et al. FAROES: Fairness And Reliability using Overlay Expenseless Set-out for duty-cycle optimization in WSN. 2011.

16. Aliouat, Z. and Z. Aliouat. Improved WSN Life Time Duration through Adaptive Clustering, Duty Cycling and Sink Mobility. in International Conference on Information Management and Engineering. 2016.

17. Ringwald, $\mathrm{M}$. and $\mathrm{K}$. Romer. Practical time synchronization for Bluetooth Scatternets. in International Conference on Broadband Communications, Networks and Systems, 2007. Broadnets. 2007.

18. Wâhslén, J., I. Orhan, and T. Lindh. Local Time Synchronization in Bluetooth Piconets for Data Fusion Using Mobile Phones. in International Conference on Body Sensor Networks. 2011. 